\title{
Modeling and Tissue Parameter Extraction Challenges for Free Space Broadband fNIR Brain Imaging Systems
}

\author{
E. Sultan ${ }^{\mathrm{a}}$, K. Manseta ${ }^{\mathrm{a}}$, A. Khwaja ${ }^{\mathrm{a}}$, L. Najafizadeh ${ }^{\mathrm{c}}$, A. Gandjbakhche ${ }^{\mathrm{c}}$, K. Pourrezaei ${ }^{\mathrm{b}}$, \\ A.S. Daryoush ${ }^{* a}$ \\ ${ }^{a}$ Department of ECE, Drexel University, Philadelphia, PA 19104 USA \\ ${ }^{\mathrm{b}}$ School of Biomedical Engineering and Health Systems, Drexel University, Philadelphia, \\ Pennsylvania 19104, USA \\ ${ }^{c}$ National Institutes of Health, 9000 Rockville Pike, Bethesda, Maryland 20892 USA \\ *daryoush@coe.drexel.edu; 12158952362
}

\begin{abstract}
Fiber based functional near infra-red (fNIR) spectroscopy has been considered as a cost effective imaging modality. To achieve a better spatial resolution and greater accuracy in extraction of the optical parameters (i.e., $\mu_{\mathrm{a}}$ and $\mu_{\mathrm{s}}$ ), broadband frequency modulated systems covering multi-octave frequencies of $10-1000 \mathrm{MHz}$ is considered. A helmet mounted broadband free space fNIR system is considered as significant improvement over bulky commercial fiber fNIR realizations that are inherently uncomfortable and dispersive for broadband operation. Accurate measurements of amplitude and phase of the frequency modulated NIR signals $(670 \mathrm{~nm}, 795 \mathrm{~nm}$, and $850 \mathrm{~nm})$ is reported here using free space optical transmitters and receivers realized in a small size and low cost modules. The tri-wavelength optical transmitter is based on vertical cavity semiconductor lasers (VCSEL), whereas the sensitive optical receiver is based on either PIN or APD photodiodes combined with transimpedance amplifiers. This paper also has considered brain phantoms to perform optical parameter extraction experiments using broadband modulated light for separations of up to $5 \mathrm{~cm}$. Analytical models for predicting forward (transmittance) and backward (reflectance) scattering of modulated photons in diffused media has been modeled using Diffusion Equation (DE). The robustness of the DE modeling and parameter extraction algorithm was studied by experimental verification of multi-layer diffused media phantoms. In particular, comparison between analytical and experimental models for narrow band and broadband has been performed to analyze the advantages of our broadband fNIR system.
\end{abstract}

\section{Introduction}

Traumatic brain injury (TBI) is a neurological disorder that in most cases caused by head injuries caused by blast injuries $^{1}$ or head on collisions ${ }^{2}$. In particular, impact acoustic pressure waves or jerking of brain in skull results in mild or severe traumatic brain injuries. For earlier diagnosis of TBI, functional brain mappings need to be performed on patients shortly after the head injuries. In most situation access to sophisticated functional Magnetic resonance imaging (fMRI) scanners are not available in field; moreover, understanding of the TBI could be developed when real time brain functionality could be compared against calibrated baseline performance. To accomplish this goal, a field transportable and low cost imaging instruments is envisioned using functional near IR (fNIR) imaging systems. Since better understanding of the brain functionality is tied to assessment of the electrical impulses (i.e., Action Potentials) ${ }^{3}$, as the main method for the nerve system to communicate and propagate with cells and organs. The action potentials are generated when certain level of energy is consumed in neurons by absorbing oxygen. Knowing the rate of oxygenated and deoxygenated blood circulation in to various regions of brain will lead to neurons and brain functionality. Imaging techniques based on absorption and scattering ${ }^{4}$ of light at near-infrared region emitted from lasers and collected by optical detectors give an accurate assessment about brain functionality. Different wavelengths in near IR region have different absorption and scattering properties for hemoglobin. Knowing the scattering and absorption coefficients will provide information about the level of oxygen absorption and hence the action potential activity associated with a

Imaging, Manipulation, and Analysis of Biomolecules, Cells, and Tissues IX, edited by Daniel L. Farkas, Dan V. Nicolau, Robert C. Leif, Proc. of SPIE Vol. 7902, 790223 - (c) 2011 SPIE · CCC code: 1605-7422/11/\$18 · doi: 10.1117/12.875618 
segment of brain. This information collected in various regions of brain will provide level of oxygen provided to neurons as a function of time.

Spectroscopic studies of tissue have shown absorption and scattering sensitivity of water, oxygenated, and deoxygenated hemoglobin at different wavelengths ${ }^{5}$. Therefore, any impact that will lead to disorder the functionally of the neurons of brain could then be registered by disorder in the absorption of oxygenated and de-oxygenated hemoglobin using diffused photon NIR (DPNIR). The location and percentage of oxygen absorbed in the brain can be related to different physiological activities ${ }^{6}$ and hence labeled as fNIR system. Knowing the level of oxygen in blood would help in early treatment of any biological disorder. Therefore localization hematoma could be made by measuring reduction in de-oxygenated hemoglobin and increase in oxygenated blood volume and having this localized information related to TBI. There are many challenges that concern early diagnose and early treatment as part of the external treatment of the human body. Since the demand for oxygenated hemoglobin increases with hematoma, while due to brain injury functionality of brain is impacted and a reduction in de-oxygenation of blood in certain areas of the brain is experienced after blast compared to the baseline behavior before. Moreover, efficacy of various proposed treatment could be compared by monitoring performance of brain regions over time.

There are different types of spectroscopy that can provide information related to brain functionality. Even though fMRI can provide an accurate assessment for brain activity ${ }^{3}$ but it cannot determine if the activity is related to a benign or malignant tumors ${ }^{7}$. However, fNIR can give information related to amount of absorption of different wavelength in different matters, but because of photon limited path migration then it becomes difficult to provide information for deep brain activity. There have been studies depicting a relation between surface spectroscopy and deep brain activity ${ }^{8}$. Functional spectroscopic measurements of brain tissue at NIR wavelengths of $670 \mathrm{~nm}, 795 \mathrm{~nm}, 850 \mathrm{~nm}$ can be used to accurately detect levels of oxygenated and deoxygenated hemoglobin as depicted in Fig $1^{9}$ from light absorption and scattering. These three wavelength will provide information related to absorption of photon in oxygenated and deoxygenated hemoglobin.

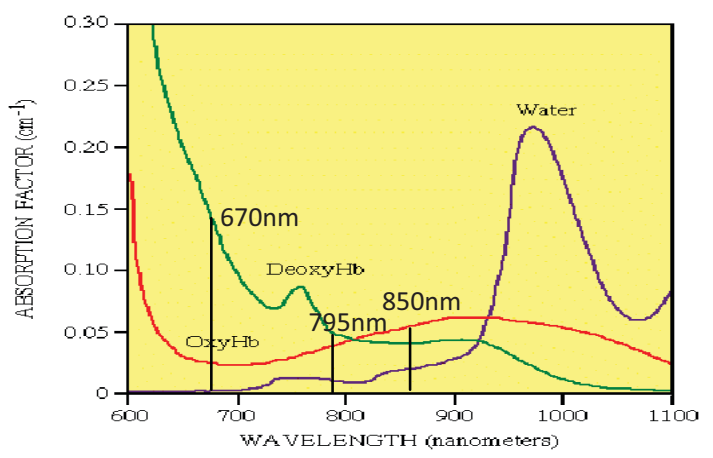

Fig. 1. NIR Spectrum with absorption coeffieints.

The fNIR imaging system is designed using multi-wavelength optical sources that are strategically located on a helmet mounted system as shown in Fig. 2. A completely mobile and field deployable unit requires a broadband and low power consuming wireless system to communicate between the sensor and the remote monitoring device. This system will be based on free space which will eliminate the use of bulky optical fibers. VCSEL, vertical-cavity surface-emitting laser, is semiconductor laser diode with laser beam emission perpendicular from the top surface which can be used as a photon source. Optical receivers using low noise PIN photodiodes or APD are employed for light collection. A relatively low level of signal processing functions are to be performed at the helmet mounted electronics to reduce the extremely high raw information throughput. The received signals over $10-1000 \mathrm{MHz}$ from optical receivers are processed locally to reduce significantly data throughput from $\mathrm{Tb} / \mathrm{s}$ to $\mathrm{Mb} / \mathrm{s}^{10}$. The block diagram for the wireless system is shown in Fig. 3 . The wireless system consists of transmit and receive section. In the receive section, the wireless signal is received by an antenna, amplified using a Low Noise Amplifier and then down converted to IF signal using a Gilbert Cell Mixer. The IF signal is then amplified using Drive Amplifier and sent as the modulating signal to the optical transmitter. For the 
transmit section, the signal from the optical receiver is sent to a Virtex FPGA processing chip, where the data throughput of the system is reduced significantly. The low throughput signal is then up converted using a Gilbert Cell Mixer and then after amplification by a Drive Amplifier, transmitted wirelessly using integrated fractal antenna. The expected path loss is $40 \mathrm{~dB}$ and thus to overcome this loss, we need the transmitter and receiver gain to be around $25 \mathrm{~dB}$. The amplifier and mixer is designed using 90nm CMOS technology from IBM. The LNA has $20 \mathrm{~dB}$ gain and a Noise Figure of $3 \mathrm{~dB}$. The GCM has a conversion gain of $4 \mathrm{~dB}$ with Noise Figure of $4 \mathrm{~dB}$. The total power consumption for the amplifier is $36 \mathrm{~mW}$ and for the mixer is $2.3 \mathrm{~mW}$. Total power consumption of the wireless part is expected around $1 \mathrm{~W}$. Assuming a battery life cycle of $3000 \mathrm{mAH}$, the total life span of the battery before charging is expected to be around 1 hour. All the optical transmitters and optical receivers can be placed on a helmet mounted system which is completely untethered. The wireless transmitter and receiver are also placed on the helmet, thus leading to a completely mobile and untethered FNIR Brain Imaging device.

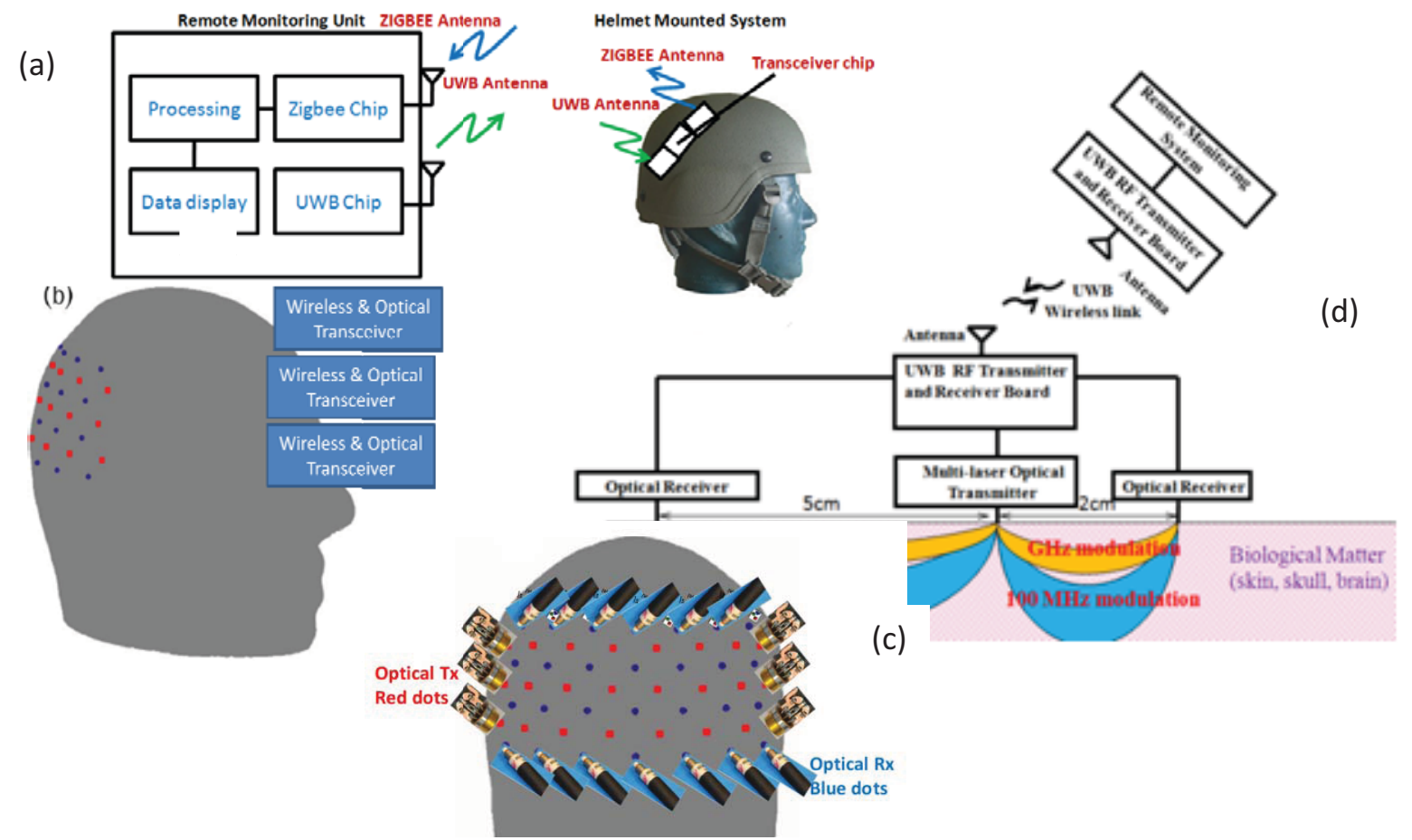

Fig. 2. Conceptual rendering of un-tethered fNIR brain imaging of TBI. a) Helmet structure used for closed TBI assessment with radiating antenna for wireless connection to a remote monitoring system. b) Proposed location and placement of wireless and optical transmitter/receiver (transceiver) modules on head. c) Physical rendering of small size optical transmitter (Tx) in red dots and optical receiver (Rx) in blue dots, where Tx and Rx are in contact with skin using quartz optical windows in free space optics as opposed to bulky and position sensitive fat optical fibers. d) Comparison of frequency modulated of DPNIR photon flow (i.e., banana shaped structure) in turbid medium of head at low (100 MHz) and high (GHz) frequencies at two separation distances of $2 \mathrm{~cm}$ and $5 \mathrm{~cm}$ between optical Tx and Rx modules of (c). Interface circuits in block diagram also highlighting ultra wideband (UWB) wireless communications between helmet and remote monitoring system. 


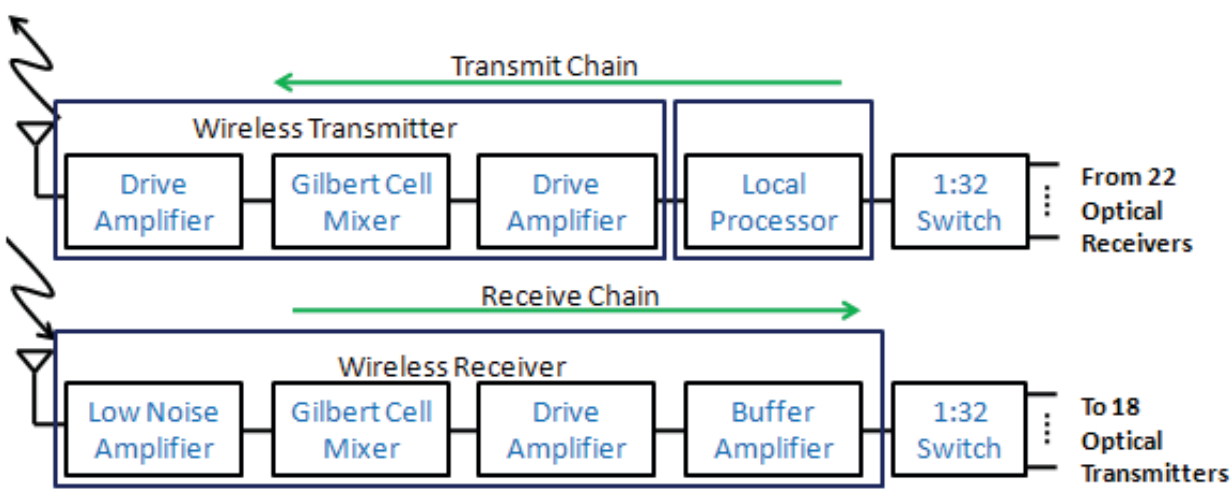

Fig.3. Block Diagram of the wireless communication system.

This paper focus on free space broadband frequency parameter extraction techniques and signal processing functions that is required. There are several advantages of broadband frequency over narrowband frequency measurements. Since most tissues have a multi layered structure and because photon penetration depth is less at higher frequency, by sweeping modulation frequency one can have information for all layers in a single measurement. This approach will give more accurate results in clinical measurements, where accuracy is essential for better diagnosis. The better assessments in parameter extraction will be used in the future to develop a helmet shape fNIR system Fig. 2. That will be controlled by remote monitoring unit.

\section{2. fNIR Background}

\subsection{Tissue Parameter Extraction}

Analytical solution to the photon transport equation ${ }^{9}$ that predicts spatial and temporal evolution of photons through biological matter is of great interest to solve inverse problem of physiological extraction that is used for biomedical imaging. In many cases an exact solution of the transport equation is not possible and instead a variety of approximations have been used. Various methods are reported of describing the photon movements/migration in any turbid medium ${ }^{11,12 \text {, }}$ 13 among them Monte Carlo (MC) simulation would give an approximation for the transport equation ${ }^{13,14}$. The basic premise of MC simulation is that complex particle- biological matter interactions can be treated as stochastic process, with simulated random samples from probability density functions. The computational tool required for inverse problem solution using $\mathrm{MC}$ technique is computationally prohibitive for complex structures such as brain. Therefore, alternative modeling technique using simplified diffusion equation (DE) to model photon migration through tissue by explaining absorption and scattering coefficients. It has been demonstrated that the DE could predict a behavior as accurate as MC technique for modulation frequencies up to $1 \mathrm{GHz}{ }^{15}$.The extracted parameters describe the hemodynamic response of human physiological organs with reported clinical applications in breast cancer detection; wound healing; and many others. The diffusion equation has been used in our analysis for the broadband analytical extraction ${ }^{16}$.

The diffusion equation states:

$$
\frac{1}{c} \frac{\partial \phi(r, \hat{s}, t)}{\partial t}-D \nabla^{2} \phi(r, t)=-\left(\mu_{a}\right) \phi(r, t)+S(r, \hat{s}, t)
$$

and we are interested in the solution of $\phi(r, t)$ for reflectance or transmittance mode. For example solutions of DE for a homogenous medium as shown in Fig. 4 can be described as a function of separation between optical source and optical detector. Since we are interested in clinical application then only reflectance mode is what matter from our analysis. The PDW is expressed for a sinusoidal point source modulated at angular frequency of $\omega=2 \pi f$ in a semi-infinite medium as $^{9,17}$ :

$$
\left.\emptyset(r, t)=\frac{A_{d c}}{4 \pi D} \frac{\exp \left(-\frac{r}{\delta}\right)}{r}+\frac{A_{a c}}{4 \pi D} \frac{\exp \left(-k_{\text {real }} r\right)}{r} \times \exp \left[-i\left(k_{\text {imag }} r-\omega t\right)\right)\right]
$$


where $A_{d c}$ and $A_{a c}$ are respectively the DC and RF components of the source, $\delta$ is the DC penetration depth, and $k_{\text {real }}$ and $k_{\text {imag }}$ are the real and imaginary components of PDW complex wave number. Therefore reflectance mode solution will be

where

$$
\begin{aligned}
& \theta(\rho, \omega)=K_{\text {imag }}(\omega) r_{o}-\arctan \left(\frac{\text { Imag }}{\text { Real }}\right) \\
& A_{\text {att }}(\rho, \omega)=\frac{\text { Air }}{4 \pi D \rho}\left(\text { Real }^{2}+\operatorname{Imag}^{2}\right)^{\frac{1}{2}}
\end{aligned}
$$

$$
\begin{aligned}
& r_{o}=\left[\left(\mu_{s}\right)^{-2}+r^{2}\right]^{\frac{1}{2}} \\
& r_{o b}=\left[\left[\frac{4}{3 \mu_{s}} \frac{1+R_{e f f}}{1-R_{e f f}}+\frac{1}{\mu_{s}}\right]^{2}+r^{2}\right] \\
& K_{\text {real }}=\sqrt{\frac{3}{2} \mu_{a} \mu_{s}^{\prime}}\left\{\left[1+\left(\frac{\omega}{c \mu_{a}}\right)^{2}\right]^{\frac{1}{2}}+1\right\}^{\frac{1}{2}} \\
& K_{\text {real }}=\sqrt{\frac{3}{2} \mu_{a} \mu_{s}^{\prime}}\left\{\left[1+\left(\frac{\omega}{c \mu_{a}}\right)^{2}\right]^{\frac{1}{2}}-1\right\}^{\frac{1}{2}} \\
& \text { Real }=\frac{\exp \left[-K_{\text {real }} \times r_{o}\right]}{r_{o}}-\cos \left[K_{\text {imag }}(\omega)\left(r_{o b}-r_{o}\right)\right] \frac{\exp \left[-K_{\text {real }}(\omega) \times r_{o b}\right]}{r_{o b}} \\
& \text { Imag }=\sin \left[\left[K_{\text {imag }}(\omega)\left(r_{o b}-r_{o}\right)\right] \frac{\exp \left[-K_{\text {real }}(\omega) \times r_{o b}\right]}{r_{o b}}\right]
\end{aligned}
$$

c is the speed of light in the medium, $\mathrm{D}$ is the scattering distribution $D=1 /\left(3 \cdot \mu_{S}^{\prime}\right)$ and $\rho$ is the separation distance between transmitter and receiver.

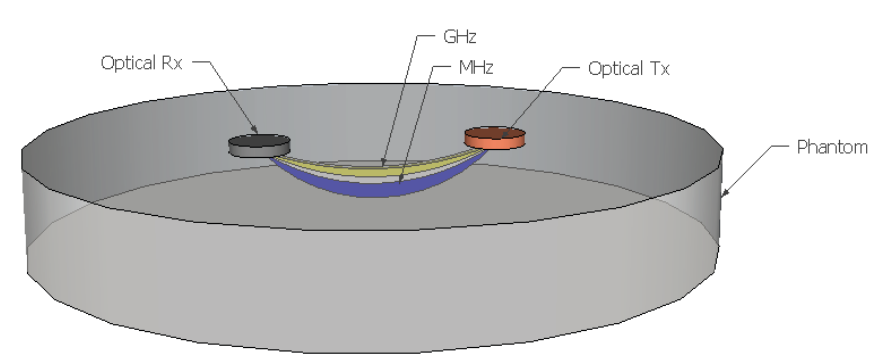

Fig.4 . Phantom Reflectance mode setup

The DE can be used for both time and frequency domain analysis. The frequency domain depends on the frequency modulation of the photons. This method have been used to investigate the optical propertied of biological matter, and both single frequency and broadband modulation technique was implemented. bandwidths of $100 \mathrm{MHz}{ }^{18,19}, 400 \mathrm{MHz}^{20}$, and $600 \mathrm{MHz}^{21}$ showed a better accuracy of parameter extraction of $\mu_{a}$ and $\mu_{s}^{\prime}$. These studies used either the insertion loss or the insertion phase information to extract the optical parameter. Therefore the information associated with high frequency response is limited and would not contain full frequency response information. The extraction of accurate absorption and scattering coefficients for biological matter turbid media can be enhanced through the use of broadband frequency-domain measurements. It is proposed that broadband frequency modulation would give a better and more accurate assessment for optical parameter extraction. Studies show ${ }^{22,23}$ that broadband modulation (DC-1GHz) would give a high accuracy when both malignant and benign tumor is present. These studies were based on fiber optical system, and would increase the noise due to the patient movement. Therefore a practical solution would eliminate the bulky fiber to enhance the information and eliminate the noise artifact due to human discomfort induced movements. 
Parameters accuracy is the major concern for any medical case because it gives better assessment of the tumor/disorder/traumatic behavior. The higher the oxygen absorption the more it become risky and indicates high level of activity that can be related to tumor/disorder/traumatic behavior. Therefore absorption coefficient need to be accurate for different NIR spectroscopy. It have been shown that broadband modulation up to $1 \mathrm{GHz}$ give a very accurate measurements for fiber optic based system ${ }^{22}$. Even though single frequency modulation gives a relative result but significant parameter accuracy is not achieved, when there are potential deviation between predictions based on true values of $\mu_{a}$ and $\mu_{s}^{\prime}$ and measurement results. In this paper we will investigate the accuracy of optical parameter extraction for free space frequency domain modulation. Our focus is in broadband systems since it permits our extraction of multilayer diffused media as encountered in head with skin, skull, CSF, and brain matter layers present.

\subsection{Review of Reported fNIR Hardware}

There are a number of commercially available fNIR systems in the market. Some uses CW, such as OTIS-2 fNIR system developed by Archinoetics ${ }^{24}$, LLC, fNIR Imager from fNIR devices, and BIOPAC ${ }^{25}$ Systems, Inc. CW system depends on constant intensity of infrared light during the measurement period. A portable three wavelength time resolved spectroscopy system from Hamamatsu is also available in the market. Model TRS- $10^{26}$ is controlled by a computer for pulsed light source at three wavelengths $(761,795$, and $835 \mathrm{~nm})$ and photon counting head for single photon detection. Honda have announced in 2009 that they have developed the world's first Brain Machine Interface (BMI) technology that uses electroencephalography (EEG) and near-infrared spectroscopy (NIR). All the commercially available products use tethered fiber optic based system. Custom designed systems have also been developed by various research teams depending on each field of interest and application. The developed systems are summarized in table 1.

\begin{tabular}{|c|c|}
\hline System type & Research Group \\
\hline Time Domain & $\begin{array}{l}\text { 1. University of Munchen, Prof. H Heusmann lab } \\
\text { 2. University of Muenster, Prof. E Haller } \\
\text { 3. University of Pennsylvania, Prof. Britton Chance } \\
\text { 3,29 }\end{array}$ \\
\hline Frequency Domain & $\begin{array}{l}\text { 1. University of California, Prof. B. Tomberg and Prof. } \\
\text { E Gratton }^{30,31} \\
\text { 2. City University of New York, Prof. R. Alfano } \\
\text { 3. TUFTS University, Prof. S Fantini } \\
\text { 31,32 }\end{array}$ \\
\hline $\mathrm{CW}$ & $\begin{array}{l}\text { 1. Rensselaer Polytechnic Institute, Prof. M Schoelles }{ }^{33} \\
\text { 2. Drexel University, Prof. Pourrezaei }{ }^{34}\end{array}$ \\
\hline
\end{tabular}

Narrow band frequency modulation, $\mathrm{CW}$, and time domain technique has limited accuracy when it come to optical parameter extraction. Broadband frequency modulation technique would give information related to deferent penetration depth and present high accuracy for optical parameter extraction. A relative measurement have been developed in most of the research laboratory to extract optical parameters using different analytical techniques, but free space (un-tethered) system have not been achieved. Limitation due to fiber system can be avoided by free space wireless system but more challenges come along. Challenges due to noisy environment and light collection have been investigated in this paper. Approaches such as two separation subtraction ${ }^{31}$ and GRIN lens can eliminate the artifact due to the transmitter and receiver and low light collection respectively.

\section{Module development and measurements}

\subsection{Approach}

There are two components that need to be considered when the optical path length modeling is being developed. The first is the optical transmitter and second is the optical receiver. The optical transmitter would provide modulating photons to penetrate into the brain matter while the optical receiver has to detect diffused and scattered photons that carry localized action potential information from brain matter. Various options for optical transmitter sources would be GaAs based 
LEDs, Edge Emitting Lasers, and Vertical Cavity Surface Emitting Lasers (VCSEL). VCSEL has more advantages over the other two edge emitting structures, because of its lower threshold current and its circular Guassian beam radiation as compared to elliptical beam in the other two geometries. In order to perform spectroscopy more than one wavelength has to be modulated at a time; therefore, a single pole four throw, SP4T, switch have been considered in case of using four wavelengths. Since free space spectroscopy is desired then light Collection of scattered light in free space system is being addressed using efficient concentration of light using GRIN lens structures. The light traveling out of the diffused medium is incident at multiple random angles. Thus to improve the collection efficiency and to have maximum coupling between the diffused medium and the optical receiver, we need a focusing device at the surface of the diffused medium. Large diameter optical fibers can be used for high collection efficiency, however they suffer from severe modal dispersion for wavelengths of $680 \mathrm{~nm}$ to $980 \mathrm{~nm}$. To overcome this problem, we propose to use GRIN lens at the Optical Receiver. The GRIN lens selected from Nippon Sheet Glass has a diameter of $1.8 \mathrm{~mm}$ and a length of $9.8 \mathrm{~mm}$ corresponding to a 0.47 pitch GRIN lens. A GRIN lens employed at $795 \mathrm{~nm}$ wavelength has a coupling efficiency of $89 \%$ as compared to $30 \%$ coupling efficiency of a $1 \mathrm{~mm}$ plastic optical fiber. However the GRIN lens is very selective to placement and minor misplacements will lead to higher losses as compared to the plastic optical fiber.

The GRIN lens are integrated with optical receiver, which are Si based PIN photodiode or Si APD. PIN photodiode has far lower DC biasing requirements than APD, which is an important specification when electrical shocks are to be avoided. However, APD has internal multiplication gain, while PIN photodiode does not. However, this internal gain of APD results in increased in noise due to random avalanche multiplication factor and is manifested as excess noise. To properly amplify the output signal from PIN Photodiode, trans-impedance amplifier is required to meet signal levels observed in the case of APD. Variable trans-impedance amplifier gains are required to meet the required signal level over the measurement system noise floor. However, the received optical signals are amplified and converted to voltage before being digitized by analog-to-digital convertor (ADC) in order to perform local signal processing in a system on a chip required prior to wireless transmission to remote unit. Our proposed design approach is based on custom developed four wavelengths VCSEL packaged in a small TO-38 can. On the optical receiver, a PIN photodiode with built in TZA and GRIN lens are being developed to accommodate the overall signal collection efficiency and amplification.

\subsection{Free space Transmitter and receiver design}

The overall measurement system block diagram of a multi wavelength NIR spectroscopy system is shown in Fig. 5. As shown in Fig. 6 a multi wavelength source have been implemented. Tri-wavelength VCSEL is commercially available from VIXAR (Module V3WLM-001) with pin designations for three wavelength of interest as depicted in Table 2. This module provide three wavelength in the near infra red region $670 \mathrm{~nm}, 795 \mathrm{~nm}$, and $850 \mathrm{~nm}$. It's a high power lasers that has a threshold current of $8.5 \mathrm{~mA}$ for the $670 \mathrm{~nm}, 9.5 \mathrm{~mA}$ for the $795 \mathrm{~nm}$, and $2.8 \mathrm{~mA}$ for the $850 \mathrm{~nm}$.

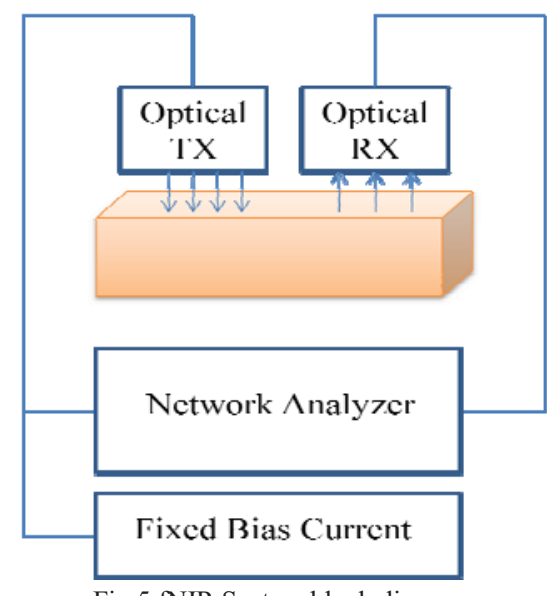

Fig.5 fNIR System block diagram 


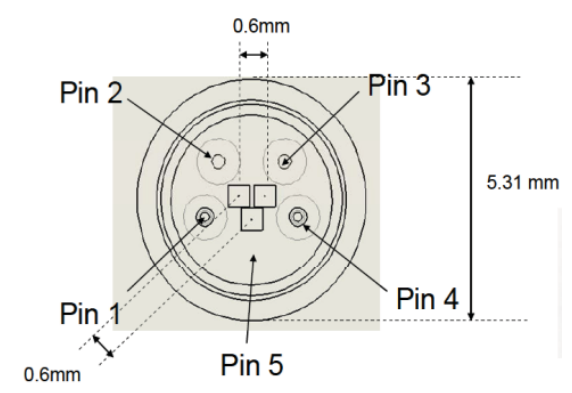

Table.2. Pin Description for VIXAR Tri-wavelength VCSEL

Fig.6. Top view schematic of the Tri-wavelength VIXAR

\begin{tabular}{|c|c|}
\hline & Pin Description \\
\hline Pin 1 & Anode - 670 VCSEL \\
\hline Pin 2 & Anode - 795 VCSEL \\
\hline Pin 3 & Anode - 850 VCSEL \\
\hline Pin 5 & Cathode All VCSELs \\
\hline
\end{tabular}

Automatic Network Analyzer (Anritsu MS4623B) is used as RF source and a sensitive RF receiver. RF switch is used to drive different high power VCSEL $(670 \mathrm{~nm}, 795 \mathrm{~nm}, 850 \mathrm{~nm})$. Multi wavelength VCSEL from VIXAR (Module V3WLM-002) have been used with an SP3T RF switch from Hittite (HMC245QS16) as depicted in Fig 7. Using FR4 substrate a printed board have been design and fabricated to accommodate all components. In absence of commercial high gain optical receivers using PIN Photodiode, APD, Fig.8, from Hamamatsu (ADP module C5658) have been used along with a built in Trans-impedance amplifier. This module provide high sensitive optical measurements with spectral response between $400-1000 \mathrm{~nm}$. The built in TIA gives $34 \mathrm{~dB}$ of gain up to $1 \mathrm{GHz}$.

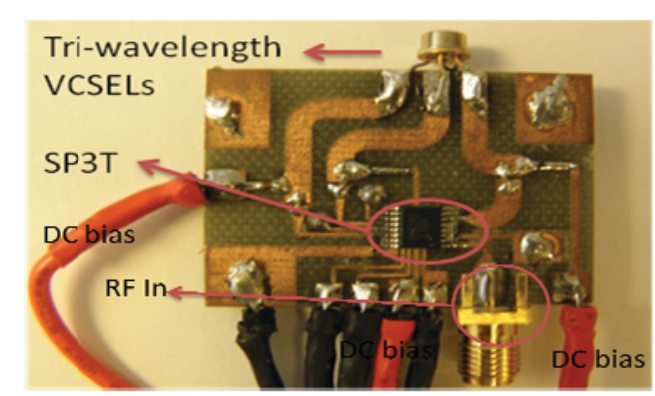

Fig. 7. Tri-wavelength laser Transmitted with single pull triple throw (SP3T) switch. Size of TO-49 can is under $6 \mathrm{~mm}$ in diameter. PCB is $3 \mathrm{cmx} 5 \mathrm{~cm}$.

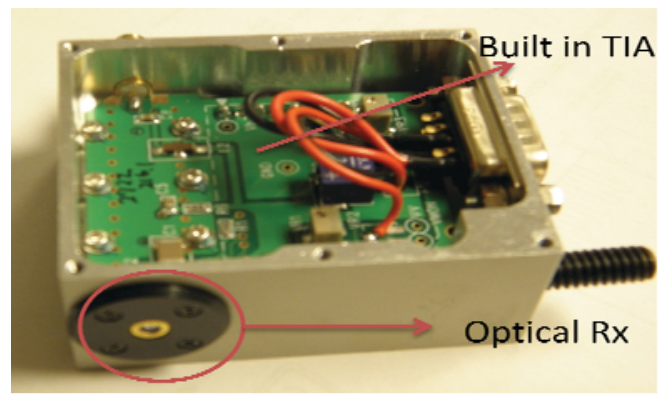

Fig. 8. Optical Receiver APD from Hamamatsu (ADP module C5658).

Signal to Noise ratio (SNR) of the optical link through diffused media of brain (i.e., Cortex, Skull, CSF) is calculated ${ }^{35}$ for different optical receivers and are depicted in Fig. 9, where shows an advantage of using PIN photodiode over an APD. A $25 \mathrm{~dB}$ difference in SNR between PIN with TZA and APD with TZA is predicted. This modeling approach is based on models reported by Daryoush et al ${ }^{36}$ 


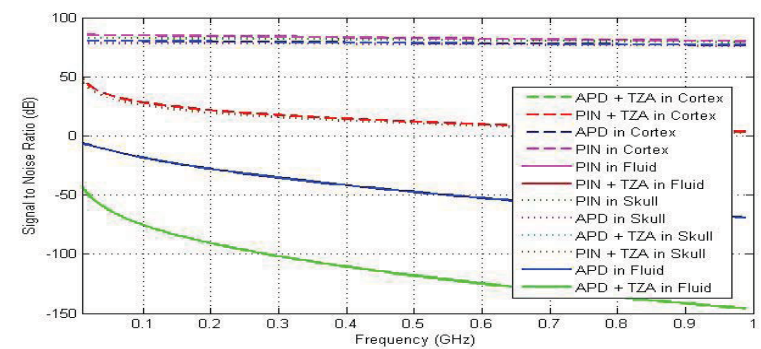

Fig. 9. Comparison of SNR for different optical receivers using PIN photodiode and APD.

\subsection{Phantom Model for Brain}

Solid and liquid Phantoms have been used as a representative of Scalp, Skull, CSF, and Cortex. Initial values for Scalp, Skull, CSF, and Cortex have been provided using MC simulation provided from study by Hoshi ${ }^{6}$. Multilayer solid phantoms need to be designed based on the optical parameter provided in Table 3 in order to experimentally validate the current predictions and demonstrate advantages of broadband frequency modulation results for more accurate parameter extraction. A homogenous phantom depicted in Fig. 10 is being used in this study with $\mu_{a}=0.045 \mathrm{~cm}^{-1}$ and $\mu_{s}^{\prime}=10 \mathrm{~cm}^{-1}$ at $850 \mathrm{~nm}$ to resemble brain tissue.

Table.3. Initial values of optical parameter

\begin{tabular}{|c|c|c|}
\hline & $\mu_{a}\left(\mathrm{~cm}^{-1}\right)$ & $\mu_{s}^{\prime}\left(\mathrm{cm}^{-1}\right)$ \\
\hline Scalp & 0.001 & 0.15 \\
\hline Skull & 0.0008 & 0.07 \\
\hline CSF & 0.00033 & 0.01 \\
\hline Cortex & 0.002 & 0.1 \\
\hline
\end{tabular}

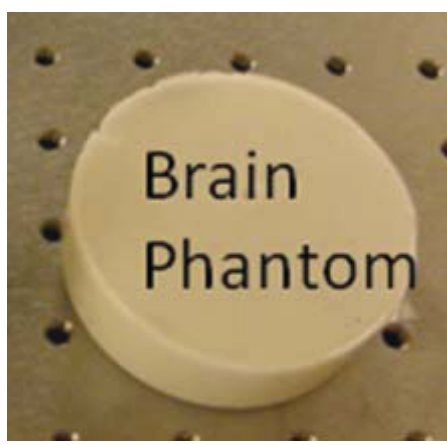

Fig.10. Brain Tissue Phantom

Using DE described in section 2.1, both the insertion loss (IL) and insertion phase (IP) have been plotted for $1 \mathrm{~cm}$ separation between the optical transmitter and the receiver for brain solid phantom shown in Fig. 10. The predicted IL and IP for different parameters associated with head are calculated and results are depicted in Fig 11a for IL and Fig. 11b for IP. that CSF have the highest insertion loss and insertion phase with respect to frequency.

(a)

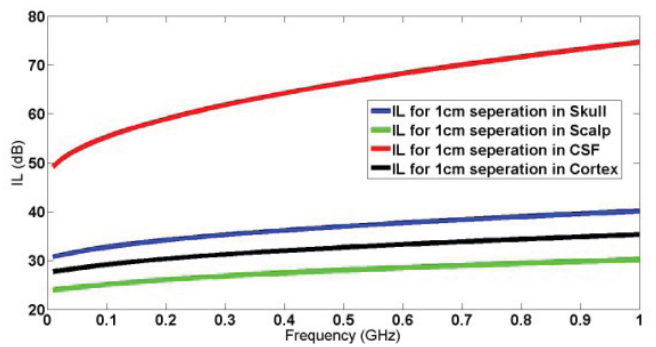

(b)

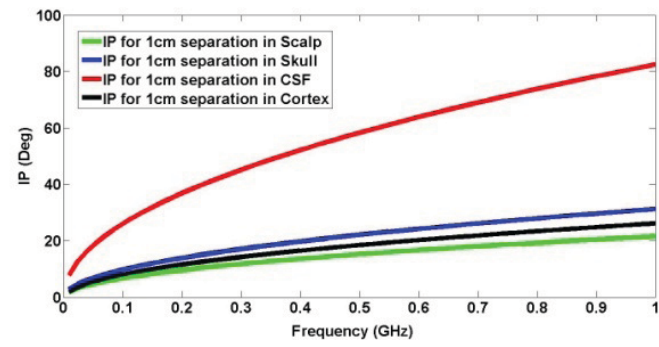

Fig.11. Predicted IL and IP for diffused density wave through Scalp, Skull, CSF, and Cortex as a function of frequency; a) IL, b) IP. 
The DE is also used to predict insertion loss and insertion phase for two separation of $1 \mathrm{~cm}$ and $1.5 \mathrm{~cm}$ for phantom that assemble brain tissue having $\quad=0.045 \mathrm{~cm}^{-1}$ and $\quad=10 \mathrm{~cm}^{-1}$ at $850 \mathrm{~nm}$. These analytical results are depicted in Fig. 12a for IL and Fig. 12b for IP and are compared later, when raw and curve fitted data discussed in the next sections.

(a)

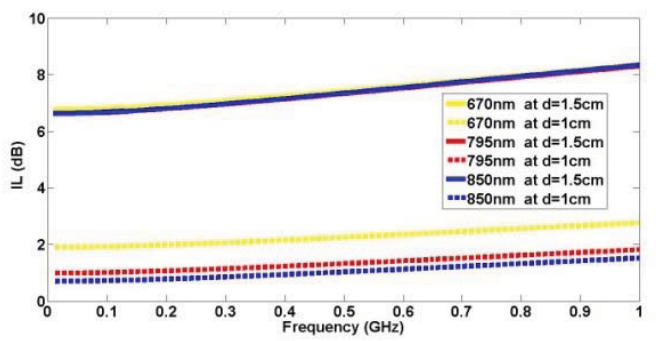

(b)

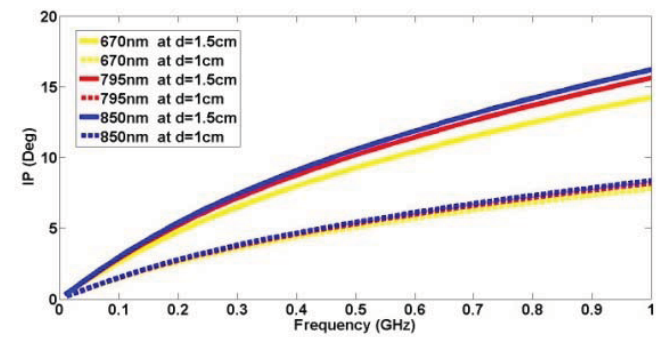

Fig. 12. Predicted IL and IP for Two separations $d_{1}=1 \mathrm{~cm}, d_{2}=1.5 \mathrm{~cm}$ as a function of frequency for three wavelengths; a) IL, b) IP.

\section{Parameter Extraction Techniques}

\subsection{Experimental Set-up for Reflectance Mode in Homogenous Media}

Uncorrelated noise sources from the optical transmitter and receiver are to be combined to quantify free space measurement noise floor level. In addition artifacts associated with frequency response limitation of a broadband laser driver and tarnsimpedance amplifier need to be removed for more accurate assessment. Two separation measurement method is proposed here to remove the artifacts of interfaces, while performing insertion loss and insertion phase measurements. The method principle is based on tissue parameter extraction by subtracting common sources of error out of two consecutive measurements at two different optical transmitter and receiver separations. In particular any errors associated with interface of optical transmitter and optical receiver with phantom is to be subtracted ${ }^{30}$. For example, the optical transmitter and receiver are placed $1 \mathrm{~cm}$ apart and insertion loss and phase are measured and stored, then the receiver is moved away by $0.5 \mathrm{~cm}$ from its original position and data are again collected. Finally mathematical operation of data/memory in ANA is performed to subtract insertion loss (in $\mathrm{dB}$ ) and insertion phase (in degrees) of these two consecutive measurements to get a result of insertion loss and insertion phase of $0.5 \mathrm{~cm}$ as depicted in Fig. 13. Total Insertion loss $(\mathrm{dB})$ of $0.5 \mathrm{~cm}$ separation is then equals Insertion loss $(\mathrm{dB})$ for $1 \mathrm{~cm}$ subtracted from Insertion loss $(\mathrm{dB})$ for $1.5 \mathrm{~cm}$, or

$$
\Delta I L(\mathrm{~dB})=I L_{d=1.5 \mathrm{~cm}}-I L_{d=1 \mathrm{~cm}}
$$

Insertion Phase (Deg) of $0.5 \mathrm{~cm}$ separation is also equals Insertion Phase (Deg) for $1 \mathrm{~cm}$ subtracted from Insertion Phase (Deg) for $1.5 \mathrm{~cm}$.

$$
\Delta I P(\mathrm{Deg})=I P_{d=1.5 \mathrm{~cm}}-I P_{d=1 \mathrm{~cm}}
$$

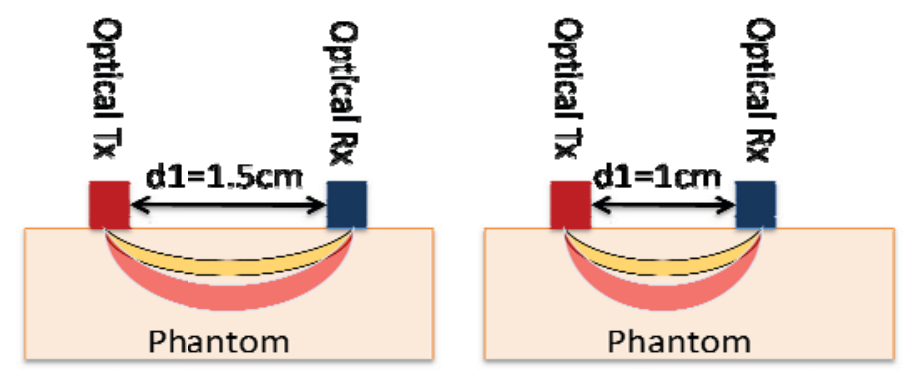

Fig. 13. Two separation method to eliminate artifact.

After implementing the optical transmitter board depicted in Fig. 7 and using the commercial optical receiver shown in Fig. 8, both insertion loss and insertion phase have been measured for two separations transmission mode analysis using backward scattering due to the solid phantom. Fig.14 shows frequency response from the Network Analyzer. 
(a)

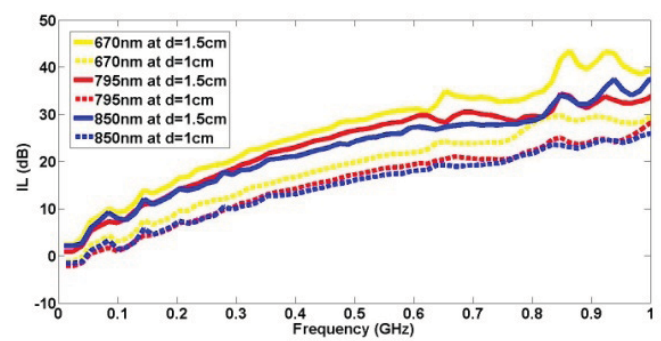

(b)

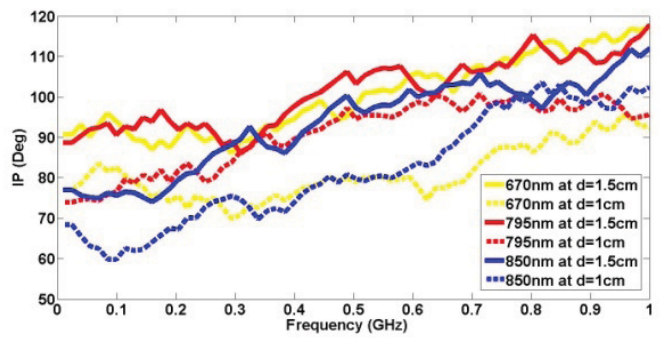

Fig.14. Absolute tri-wavelength IL and IP raw data for two separations of $1 \mathrm{~cm}$ and $1.5 \mathrm{~cm}$ through brain phantom; a) IL, b) IP.

The measurement artifact are to be subtracted using the two separation measurement techniques to get both $\Delta I L$ and $\Delta I P$ as shown in Fig.15.

(a)

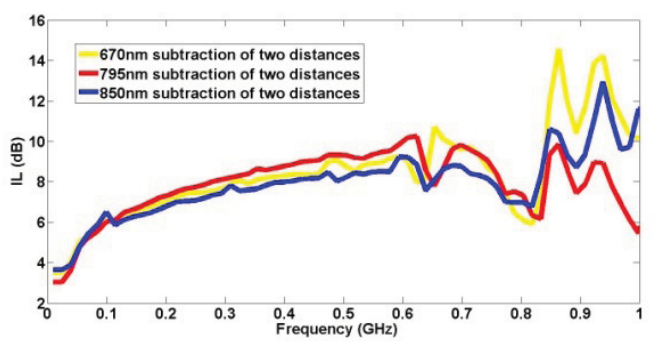

(b)

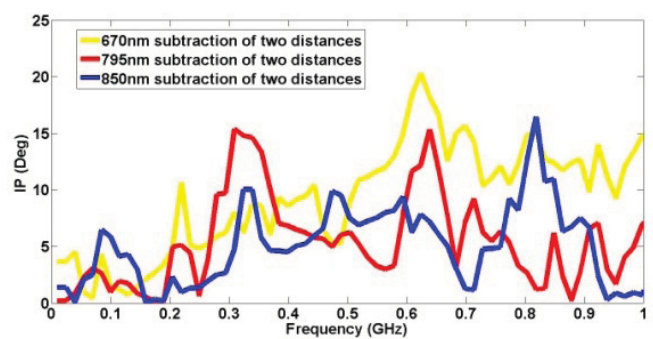

Fig.15. Relative tri-wavelength IL and IP raw data for two separations of $1 \mathrm{~cm}$ and $1.5 \mathrm{~cm}$ through brain phantom; a) $\Delta \mathrm{IL}$, b) $\Delta \mathrm{IP}$.

\subsection{Broadband Measurement and Extraction Algorithm}

Another type of signal processing would be curve fitting the data to the diffusion equation. In this step we use the diffusion equation analytical result for certain separation and then curve fit the raw data collected. Eventually, the raw data was given a similar behavior to the analytical results, but reflecting amplitude and phase fluctuation due to non-ideal behavior of the optical transmitter, inhomogeneous diffused medium, and non-ideal interface to the optical receiver. Comparison of Fig. 11a and Fig. 14a for IL and Fig. 11b and Fig. 14b for IP clearly indicates this realistic deviation from the ideal behavior. Curve fitting method of constructing mathematical function that has the best fit to a series of data points (DE).

The algorithm used for curve fitted is shown in the block diagram in Fig.16. Both insertion loss and insertion phase for two separation is curve fitted to $a \sqrt{\text { freq }}+b$, subtracted, use the least square error method in Matlab, and then extract the optical parameters. The least square curve(lsqcurvefit) fitting method is based on Levenberg-Macquart, which is used to minimize the merit function. Three wavelengths have been curve fitted using the algorithm explained in previous section. The following figures shows an advantage of using curve fitting over the raw data that can be shown by comparing these plots to the predicted IL and IP plots from section 3.3. The process starts with collecting raw data from the network analyzer which provides information about the insertion loss and phase loss. These raw data is collected from two different distances to eliminate the artifact as mentioned in previous section. After collecting the raw data a curve fitting program in matlab was created to extract the both parameter $a$ and $b$ to best fit the mathematical function of $a \sqrt{\text { freq }}+b$. Note that for each data of the three wavelengths of 670,795 , and $850 \mathrm{~nm}$, different fitting parameters are extracted to give a unique solution for each raw data as shown in Fig. 17 through Fig 19. The second step is based on subtracting the two curve fitted mathematical data to result in $\Delta I L(\mathrm{~dB})$ and $\Delta I P(D e g)$. The Insertion phase result from the curve fitted resemble the electrical loss given from the network analyzer where the diffusion equation uses the optical loss. Known that the electrical loss is double the optical loss therefore a conversion factor of 2 in $\mathrm{dB}$ values has been made. 


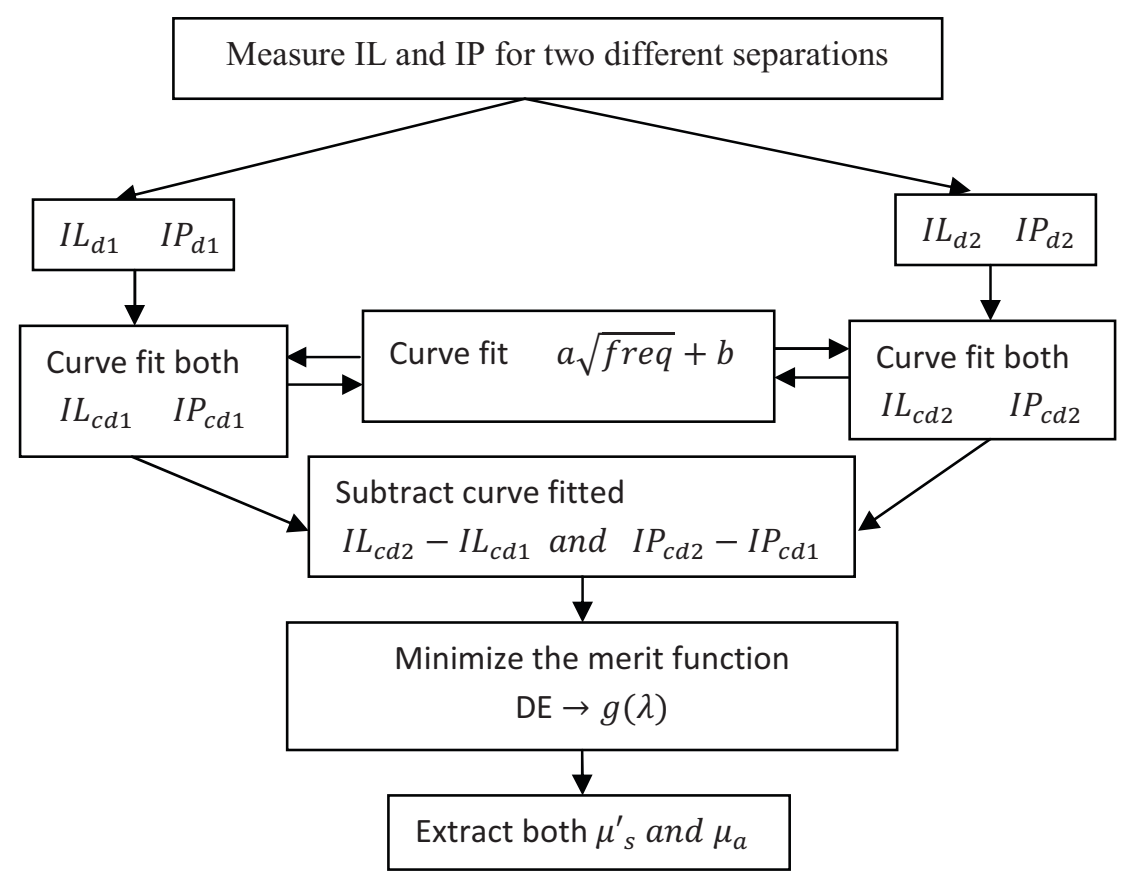

Fig. 16. Flow chart used for extraction algorithm of biological parameters.

(a)

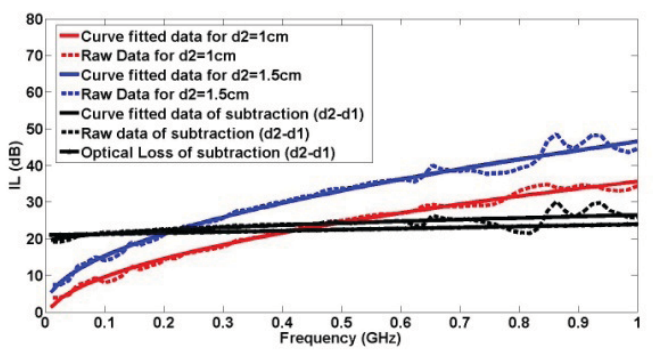

(b)

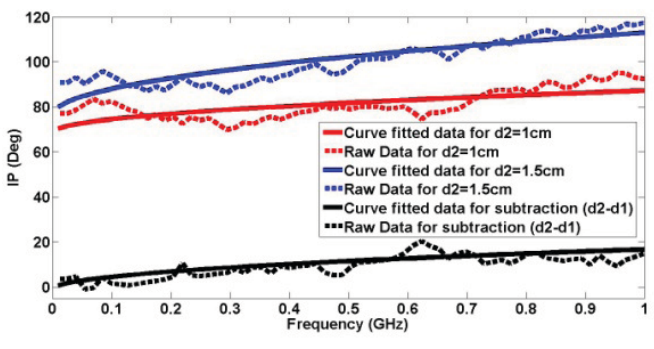

Fig. 17. Measured data, fitted, and relative IL and IP data for two separations at wavelength of $670 \mathrm{~nm}$ as a function of frequency.

(a)

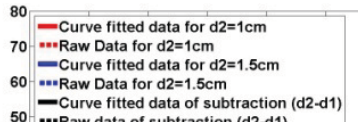

$60-\cdots$ Raw Data for $\mathrm{d} 2=1.5 \mathrm{~cm}=1$.

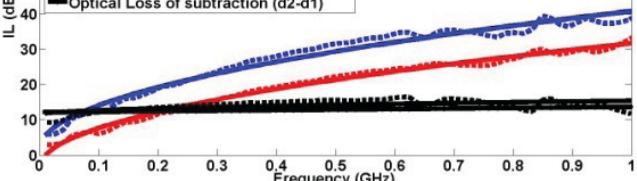

(b)

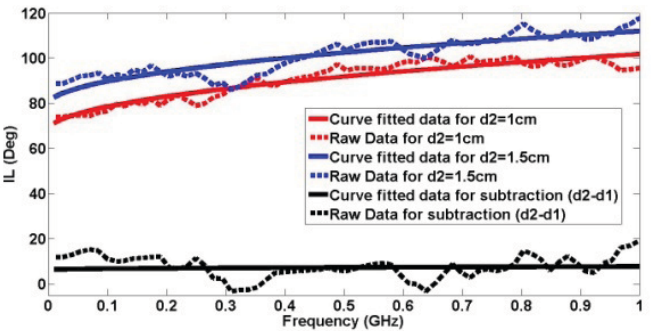

Fig. 18. Measured data, fitted, and relative IL and IP data for two separations at wavelength of $795 \mathrm{~nm}$ as a function of frequency.

(a)

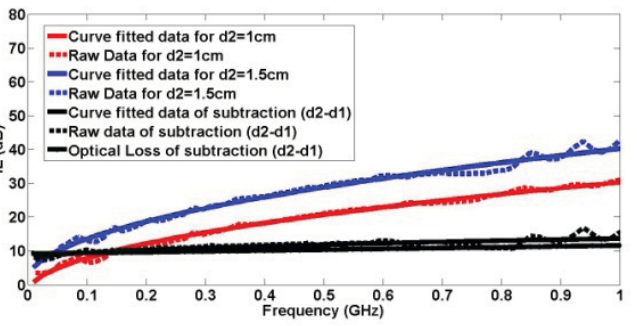

(b)

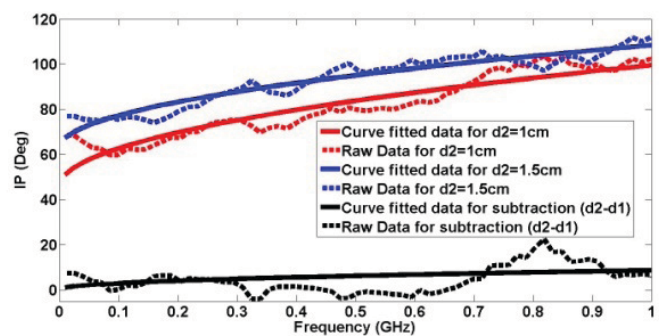

Fig. 19. Measured data, fitted, and relative IL and IP data for two separations at wavelength of $850 \mathrm{~nm}$ as a function of frequency. 
The final step is to extract the optical parameter using least square error provided from Matlab. Table 4 tabulates extracted $\mu_{s}^{\prime}$ and $\mu_{a}$ for three different wavelengths $670 \mathrm{~nm}, 795 \mathrm{~nm}$, and $850 \mathrm{~nm}$. The performance results are extracted and compared for various bandwidths and single frequencies that the fitted curve and the DE results compare very well. Note these frequencies are in the proximity of frequencies commonly reported by other groups for frequency modulated $\mathrm{I} / \mathrm{Q}$ receivers.

Table 4. Comparison between various broadband and single frequency optical parameter extraction.

\begin{tabular}{|c|c|c|c|c|c|}
\hline & & $670 \mathrm{~nm}$ & $795 \mathrm{~nm}$ & $850 \mathrm{~nm}$ & \\
\hline & g & 0.712 & 0.639 & 0.607 & $\begin{array}{l}\text { Error } \% \text { from Manufacture values at } \\
850 \mathrm{~nm}, \mu_{a}\left(\mathrm{~cm}^{-1}\right)=0.045 \mathrm{~cm}^{-1} \\
\text { and } \mu_{s}^{\prime}=10 \mathrm{~cm}^{-1}\end{array}$ \\
\hline \multirow[b]{2}{*}{$100-600 \mathrm{MHz}$} & $\mu_{s}^{\prime}\left(\mathrm{cm}^{-1}\right)$ & 13.79 & 11.57 & 10.12 & $0.6 \%$ \\
\hline & $\mu_{a}\left(\mathrm{~cm}^{-1}\right)$ & 0.025 & 0.036 & 0.0438 & $1.4 \%$ \\
\hline \multirow[b]{2}{*}{$100-1000 \mathrm{MHz}$} & $\mu_{s}^{\prime}\left(\mathrm{cm}^{-1}\right)$ & 0.042 & 0.047 & 0.158 & $55 \%$ \\
\hline & $\mu_{a}\left(\mathrm{~cm}^{-1}\right)$ & 11.93 & 8.91 & 7.2 & $16.3 \%$ \\
\hline \multirow[b]{2}{*}{ 10-100MHz } & $\mu_{s}^{\prime}\left(\mathrm{cm}^{-1}\right)$ & 0.065 & 0.01 & 0.005 & $80 \%$ \\
\hline & $\mu_{a}\left(\mathrm{~cm}^{-1}\right)$ & 14.3 & 30.84 & 24.12 & $41.4 \%$ \\
\hline \multirow[b]{2}{*}{$10-600 \mathrm{MHz}$} & $\mu_{s}^{\prime}\left(\mathrm{cm}^{-1}\right)$ & $>100$ & $>100$ & $>100$ & $>100 \%$ \\
\hline & $\mu_{a}\left(\mathrm{~cm}^{-1}\right)$ & 0.17 & 0.161 & 0.17 & $58 \%$ \\
\hline \multirow[b]{2}{*}{$10-1000 \mathrm{MHz}$} & $\mu_{s}^{\prime}\left(\mathrm{cm}^{-1}\right)$ & 13.80 & 7.15 & 7.15 & $16.6 \%$ \\
\hline & $\mu_{a}\left(\mathrm{~cm}^{-1}\right)$ & 0.030 & 0.15 & 0.15 & $54 \%$ \\
\hline \multirow[b]{2}{*}{$50 \mathrm{MHz}$} & $\mu_{s}^{\prime}\left(\mathrm{cm}^{-1}\right)$ & 19.78 & 40 & 11.56 & $7.4 \%$ \\
\hline & $\mu_{a}\left(\mathrm{~cm}^{-1}\right)$ & 0.019 & 0.0045 & 0.021 & $36.4 \%$ \\
\hline \multirow[b]{2}{*}{$141 \mathrm{MHz}$} & $\mu_{s}^{\prime}\left(\mathrm{cm}^{-1}\right)$ & 16.86 & 18.14 & 9.5 & $2.6 \%$ \\
\hline & $\mu_{a}\left(\mathrm{~cm}^{-1}\right)$ & 0.026 & 0.018 & 0.049 & $4.3 \%$ \\
\hline \multirow[b]{2}{*}{$450 \mathrm{MHz}$} & $\mu_{s}^{\prime}\left(\mathrm{cm}^{-1}\right)$ & 14.1 & 8.6 & 7.63 & $13.5 \%$ \\
\hline & $\mu_{a}\left(\mathrm{~cm}^{-1}\right)$ & 0.031 & 0.098 & 0.124 & $47 \%$ \\
\hline
\end{tabular}

Extracting the parameter was based on curve fitting different portion of the data each time. From the raw data versus curve fitted data, it was expected that the best extraction is going to be between $100 \mathrm{MHz}$ and $600 \mathrm{MHz}$, as it is highlighted with the least amount of error indicated in red in Table 4. The worst extraction in terms of percentage error is over $10-600 \mathrm{MHz}$ followed with $10-100 \mathrm{MHz}$, which is due to insertion loss and insertion Phase below $100 \mathrm{MHz}$ and above $600 \mathrm{MHz}$ are not monotonic and experience significant fluctuation. The observed fluctuations result in extraction 
error experienced in this frequency range. This is one of the main challenges of the free space and a reason for using curve fitting to minimize error. In particular our measurement from $600-1000 \mathrm{MHz}$ suffers from significant repeatable fluctuation, which its source is somewhat independent of low SNR values at those frequencies. The source of this deviation between the DE predictions and measured results is still under investigations since it is not due to experimental errors.

\section{Conclusion}

The optical link data at $670 \mathrm{~nm}, 795 \mathrm{~nm}$, and $850 \mathrm{~nm}$ has been used for this study as a representative of this signal processing based parameter extraction technique. Insertion loss increases linearly with respect to square root of frequency as shown in Fig. 17a, 18a, and 19a, whereas insertion phase is showing a linear increase with respect to frequency as depicted in Fig. 17b, 18b, and 19b. This performance is predicted since migrating photons at a higher frequency experiences a higher attenuation and phase change over a lower frequencies. The broadband and single frequency extraction of absorption and scattering parameters as a function of frequency is compared in Table 4. Only the phantom optical parameter at $850 \mathrm{~nm}$ is known from the manufacture, which indicate that the result from Table 4 at $850 \mathrm{~nm}$ is giving an error of $1.4 \%$ for $\mu_{a}$ and $0.6 \%$ for $\mu_{s}^{\prime}$. From the results, we can see that the accuracy of broadband from $100-600 \mathrm{MHz}$ with curve fitting extraction is much better than $10-1000 \mathrm{MHz}$, which excludes deviations from predicted performance using DE over $10-100 \mathrm{MHz}$ and $600-1000 \mathrm{MHz}$. Even the extraction of $\mu_{a}$ and $\mu_{s}{ }^{\prime}$ at the single frequencies that we observe the best match between the curve fitted data and raw data does not lead to the same accuracy as the broadband results. The source of deviation between the DE predictions and measured results for frequencies of $10-50 \mathrm{MHz}$ and $600-1000 \mathrm{MHz}$ is under investigations since it is not due to experimental errors.

\section{Acknowledgement}

This research is partly supported by the Center for Neuroscience and Regenerative Medicine (CNRM), and the Intramural Research Program (IRP) of Eunice Shriver National Institute of Child Health and Human Development (NICHD) of the National Institute of Health.

\section{References:}

[1] Merzagora AC, Polikar R, Schultheis MT, Onaral B, (2008). Combined fNIRS and EEG for the assessment of cognitive impairments following traumatic brain injury. Society of Applied Neuroscience Intl. Conf. 2008

[2] Irani F, Platek SM, Bunce S, Ruocco AC, Chute D. (2007). Functional Near Infrared Spectroscopy (fNIRS): An Emerging Neuroimaging Technology with Important Applications for the Study of Brain Disorders. The Clinical Neuropsychologist, 21:9-37

[3] Ojemann, G.A., Corina, D.P., Corrigan, N., Schoenfield-McNeill, J., Poliakov, A., Zamora, L.,et al., "Neuronal correlates of functional magnetic resonance imaging in human temporal cortex". Brain 133 (Pt 1), 46-59 (2010).

[4] Frédéric Bevilacqua, Dominique Piguet, Pierre Marquet, Jeffrey D. Gross, Bruce J. Tromberg, and Christian Depeursinge, "In Vivo Local Determination of Tissue Optical Properties: Applications to Human Brain", Applied Optics, Volume 38, Number 22, pp. 4939-4950 (1999).

[5] Villringer A, Dirnagl U., "Coupling of brain activity and cerebral blood flow: basis of functional neuroimaging", Cerebrovasc Brain Metab Rev. 1995 Fall;7(3):240-76.

[6] Yoko Hoshi, "Functional near-infrared optical imaging: Utility and limitations in human brain mapping", Psychophysiology, Volume 40 Issue 4, Pages $511-520$.

[7] Fishkin, J. B., Coquoz, O., Anderson, E. R., Brenner, M., Tromberg, B. J. "Frequency-domain photon migration measurements of normal and malignant tissue optical properties in a human subject". Applied Optics. 36: 10-20, (1997).

[8] Attal, Y., Bhattacharjee, M., Yelnik, J., Cottereau, B., Lefèvre, J., Okada, Y., . (2009, June). Modelling and detecting deep brain activity with MEG and EEG. IRBM-Biomed. Eng. \& Res., 30(3), 133-38

[9] M. Patterson, B. Chance, and B. C. Wilson, "Time resolved reflectance and transmittance for the non-invasive measurement of tissue optical properties," Appl. Opt. 28, 2331-2336 (1989).

[10] A.M. Khwaja et al, "UWB Wireless Link Design and Implementation Challenges in Broadband Frequency Modulated fNIR Biomedical,” IEEE Radio and Wireless Symposium, Jan. 2011 
[11] Frédéric Bevilacqua, Dominique Piguet, Pierre Marquet, Jeffrey D. Gross, Bruce J. Tromberg, and Christian Depeursinge, "In Vivo Local Determination of Tissue Optical Properties: Applications to Human Brain", Applied Optics, Volume 38, Number 22, pp. 4939-4950 (1999).

[12] J.J. Duderstadt and W.R.Martin, "Transport Theory", John Wiley \& Sons, New York (1979)

[13] Arridge, S. R.; Schweiger, M.; Hiraoka, M.; Delpy, D. T., "A finite element approach for modeling photon transport in tissue", Medical Physics, Volume 20, Number 2, March 1993, pp. 299-309.

[14] Phillip N. Reinersman and Kendall L. Carder, "Hybrid Numerical Method for Solution of the Radiative Transfer Equation in One, Two, or Three Dimensions". Applied Optics, Volume 43, Number 13, pp. 2734-2743 (2004).

[15] Joshua B. Fishkin, Sergio Fantini, Martin J. vandeVen, and Enrico Gratton, "Gigahertz photon density waves in a turbid medium: Theory and experiment", Phys. Rev. E Volume 53, pp. 2307-2319 (1996)

[16] T.H. Pham, Olivier Coquoz, J.B. Fishkin, Eric Anderson, B.J. Tromberg, "Broad bandwidth frequency domain instrument for quantitative tissue optical spectroscopy. " Review of Scientific Instruments Volume 71, Number 6 (2000)

[17] D. Contini, F. Martelli, and G. Zaccanti, "Photon migration through a turbid slab described by a model based on diffusion approximation I. Theory," Appl. Opt. 36, 4587-4599 (1997).

[18] M. Gerken and G. W. Faris, "High-accuracy optical-property measurements using a frequency domain technique," Proc. SPIE 3597, 593-600 (1999).

[19] M. Gerken, D. Godfrey, and G. W. Faris, "Application of frequency domain techniques to samples with moderate scatter," Proc. SPIE 3599, 86-92 (1999).

[20] Holboke M., Tromberg B., Li X., et, "Three-dimensional diffuse optical mammography with ultrasound localization in human subjects." J Biomed Opt 5. (2): 237-247.(2000)

[21] A. J. Berger et al., "Chemometric analysis of frequency-domain photon migration data: quantitative measurements of optical properties and chromophore concentrations in multicomponent turbid media," Applied Optics, 39(10):1659-1667 ( 2000).

[22] Tromberg, B.J., Coquoz, O., Fishkin, J.B., Pham, T., Anderson, E.R., Butler, J., Cahn, M., Gross, J.D., Venugopalan, V., Pham, D. "Non-Invasive Measurements of Breast Tissue Optical Properties Using FrequencyDomain Photon Migration”. Phil. Trans. Royal Society London B. 352, 661-668 (1997).

[23] Daryoush, A. S. (2009) RF and Microwave Photonics in Biomedical Applications, in Microwave Photonics: Devices and Applications (ed S. Iezekiel), John Wiley \& Sons, Ltd, Chichester, UK

[24] http://www.archinoetics.com/otis-fnir.php

[25] http://www.biopac.com/

[26] http://www.hamamatsu.com/

[27] H. Heusmann, J. Kolzer, and G. Mitic, "Characterization of female breast in vivo by time resolved and spectroscopic measurements in near infrared spectroscopy, " J. Biomed. Opt. 1, 425-434 (1996).

[28] E. B. de Haller, “Time-resolved transillumination and optical tomography,'”J. Biomed. Opt. 1, 7-17 (1996).

[29] B. Chance and R. R. Alfano , "Optical Tomography and Spectroscopy of Tissue: Theory, Instrumentation, Model, and Human Studies II". Proceedings of SPIE, Vol 2979 (1997)

[30] B. Chance, M. Cope, E. Gratton, N. Ramanujam, B. Tromberg, "Review Article: Phase measurement of light absorption and scatter in human tissue," Rev. Sci. Instrum. 69 (10), pp. 3457-3481 (1998).

[31] Fantini, S., Franceschini, M. A., Fishkin, J. B., Barbieri, B. \& Gratton, E,"Quantitative determination of the ab sorption spectra of chromophores in strongly scattering media: a light-emitting-diode based technique. Appl. Opt. 33, 5204-5213 (1994)

[32] Joshua B. Fishkin, Sergio Fantini, Martin J. vandeVen, and Enrico Gratton, "Gigahertz photon density waves in a turbid medium: Theory and experiment", Phys. Rev. E Volume 53, pp. 2307-2319 (1996)

[33] Y. Son, M. Guhe, B. Yazici, and M Schoelles "Human performance assessment using. fNIR," in Proc. SPIE, vol. 5797, pp. 158-169 (2005)

[34] Izzetoglu, K., Bunce, S., Onaral, B., Pourrezaei, K., \& Chance, B, "Functional optical brain imaging using nearinfrared during cognitive tasks". International Journal of Human-Computer Interaction, 17(2), 211-231. (2004).

[35] K. Manseta et al, "Development Challenges of Brain Functional Monitoring using Untethered Broadband Frequency Modulated fNIR System," IEEE Microwave Photonics proceeding, pp. $295-299$, MWP 2010, Montreal, Canada.

[36] A. S. Daryoush, E. Ackerman, N. Samant, S. Wanuga, and D. Kasemset, "Interfaces for high-speed fiber-optic links: analysis and experiment," IEEE Trans. Microwave Theory Tech., vol. 39, pp. 203 1-2044, 1-2044, 1991 